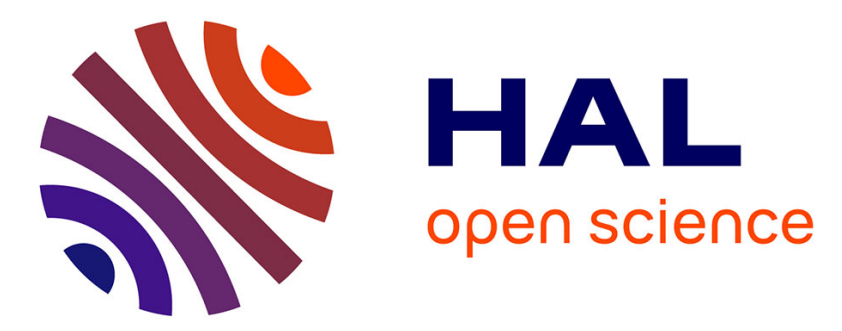

\title{
Ontology alignment for networked enterprises information systems interoperability in supply chain environment
}

\author{
Yan Lu, Hervé Panetto, Yihua Ni, Xinjian Gu
}

\section{- To cite this version:}

Yan Lu, Hervé Panetto, Yihua Ni, Xinjian Gu. Ontology alignment for networked enterprises information systems interoperability in supply chain environment. International Journal of Computer Integrated Manufacturing, 2013, 26 (1-2), pp.140-155. 10.1080/0951192X.2012.681917 . hal-00655468

\section{HAL Id: hal-00655468 \\ https://hal.science/hal-00655468}

Submitted on 29 Dec 2011

HAL is a multi-disciplinary open access archive for the deposit and dissemination of scientific research documents, whether they are published or not. The documents may come from teaching and research institutions in France or abroad, or from public or private research centers.
L'archive ouverte pluridisciplinaire HAL, est destinée au dépôt et à la diffusion de documents scientifiques de niveau recherche, publiés ou non, émanant des établissements d'enseignement et de recherche français ou étrangers, des laboratoires publics ou privés. 


\title{
Ontology Alignment for Networked Enterprises Information Systems Interoperability in Supply Chain Environment
}

\author{
Yan $\mathrm{Lu}^{\mathrm{a}, \mathrm{b}}$, Hervé Panetto ${ }^{\mathrm{b}}$, Yihua $\mathrm{Ni}^{\mathrm{a}}$, Xinjian $\mathrm{Gu}^{\mathrm{a}}$ \\ a. Institute of Manufacturing Engineering, Zhejiang University, P.R. China \\ b. CRAN UMR 7039, University of Lorraine, CNRS, France
}

Herve.Panetto@cran.uhp-nancy.fr, \{lvyan,xjgu\}@,zju.edu.cn, niyihua@hzcnc.com

Supply chain interactions are complex in general and can be viewed as a networked enterprise .In this paper, we address information system interoperability for exploring and analysing the interoperation problem in heterogeneous networked enterprises systems. We propose a common shared ontology based framework for networked enterprises interoperability for the context of information flows in supply chain interactions. A supply chain ontology, SCOR ontology, is developed based on the SCOR operation reference model. Subsequently, by formalising the similar concepts of SCOR ontology and product ontology, ONTO-PDM, using Description Logic, we propose a product-centric supply chain ontology framework for facilitating the interoperation between all enterprise applications involved in an extended supply chain. A case study of a Supply Chain Make-to-Order process is used to demonstrate the feasibility of the approach.

Keywords: ontology alignment; SCOR; supply chain; interoperability; networked enterprise; ONTO-PDM

\section{Introduction}

With the advent of global manufacturing, enterprises cannot be viewed as being isolated. Enterprise collaboration is no longer just between two partners; they have evolved to what has been described as 'enterprise networks', in forms of supply chain partnerships, extended enterprises and virtual enterprises [Jagdev, 2001]. Supply chain interactions can also be defined as a category of collaborative networks [Jagdev, 2001]. Although there has been substantial research literature on supply chain interactions, these perspectives and proposed approaches vary greatly. One common tenet is that competitive success depends on managers' ability to recognize changes in the competitive environment and then to structure organizational, and where appropriate, supply chain resources to effectively meets customers' real needs [Stanley, 2001].This is a fundamental tenet of Supply Chain Management (SCM) implementation. Meanwhile in industrial companies, rapidly developed ICT (Information and 
Communications Technology) is being adopted to handle supply chain management issues, ranging from ERP (Enterprise Resource Planning) to MPC (Manufacturing Planning and Control) and MES (Manufacturing Execution System), facilitating various aspects of the supply chain [Kollberg, 2006]. It provides a new way to store, process, distribute and exchange information both within companies and with customers and suppliers in the supply chain. This environment has increasing demands for information or knowledge exchange among enterprises. Meanwhile mass information or knowledge spread out in various formats among different enterprise systems, which lead to semantic interoperability issues between the existing enterprises information systems. The heterogeneity of these systems and the disunity of knowledge expression methods are becoming barriers for the knowledge acquisition by stakeholders. While these collaborations are being adopted, there would be excessive knowledge accumulated, unorganized and decentralized, which could lead to a considerably low efficiency and inconsistency in their treatments. All these issues will definitely affect everyone's comprehension when enterprises collaborate in the context of supply chain. In order to contribute in solving this gap both in syntactic and semantic level, ontology is currently one of the most studied paradigms. Ontology is a formal, explicit specification of a shared conceptualisation [Gruber, 1993]. It has the capability to reserve semantic meaning of knowledge through a formal representation of knowledge as a set of concepts within a domain of discourse, and the relationships between those concepts. Therefore ontology is a promising means to unify the metadata model to express knowledge resources which are diverse in types and disunited in forms.

In this paper, we give an insight into interoperability problems of networked enterprises information systems in supply chain environment. By building a new ontology with standardized concepts and information, in a reference model such as the SCOR model aligned with the ONTO-PDM Product Ontology developed by [Tursi et al., 2009], we aim at 
contributing to enterprise semantic interoperation within the supply chain context. The main perspective of this on-going work is then the definition of a product-centric supply chain ontology framework for facilitating the interoperation between all enterprises applications involved in extended supply chain interactions. The next section will present the state-of-theart in enterprise interoperability problem related works in apply ontological reference models to a Supply Chain environment and product ontology. Section 3 will give an insight on the SCOR model and the ONTO-PDM product ontology that will merge the related knowledge on Supply Chain processes and information sharing. Section 4 will apply our approach to a specific Make-to-Order process from main body of grinding machine Supply Chain case study. We will conclude our study with a summary of our contribution and some perspective for on-going and future work.

\section{Background and related works}

Supply chain management is a complicated dynamic environment consisting of organizations, people, technology, activities, information and resources all involved in moving a product or service. During more than twenty years, SCM has been trying to better configure supply chain with different goals, such as enterprise integration, outsourced manufacturing and service. To deal with mass elements and information in such a complex environment, reference model plays an important role in SCM. Several models have been proposed for supply chain process configuration. For example, SCOR promoted by the Supply Chain Council ${ }^{1}$, measures the total supply chain performance. The SCM model proposed by the Global Supply Chain Forum $(\mathrm{GSCF})^{2}$, is built on eight key business processes that are both cross-functional and cross-firm in nature. However all these models focus on supply chain process configure, and do not address any system semantic interoperation problem.

'http://www.supply-chain.org ${ }^{2} \mathrm{http}: / / \mathrm{scm}$-institute.org/ 
Nowadays, many ICT tools and IT techniques invented and involved in supply chain interaction, bring more system applications for better information management, but also they present more obstacles for systems interoperability problems. Interoperability is defined as the ability of two or more systems or components to exchange information and to use the information that has been exchanged [IEEE 1995]. It is frequent to find that information are distributed within enterprises: in applications used to manage technical data (e.g.: Product Data Management systems (PDM)), in applications that manage business information (e.g.: Enterprise Resource Planning (ERP)) and, finally, in the applications that manage manufacturing information (e.g.: Manufacturing Execution Systems (MES)). Some research works have already been done to analyse the semantic problems in supply chain and make contribution to it. Millet et al. [Millet et al, 2009] proposed an extended reference model based on SCOR operation reference model for alignment of business processes and information systems. This model is built on a multi-view of business process mapping, including the informational dimension, and thus a more practical alignment of ERP systems with processes. Sakka [Sakka et al., 2010] proposed to represent the SCOR operation reference model as an ARIS [Sheer et al., 2005] process model, and then transformed this ARIS model into a OWL format in order to add semantic into the original SCOR model. Zdravković [Zdravković, et al., 2010] proposed a SCOR model ontology for supporting knowledge management in supply chain operations. $\mathrm{SCOR}+3$ is a commercial tool directed towards overcoming the limitations of the basic SCOR model through an ontology based tools, to supply chain process definition at four levels: the supply chain level, the enterprise level, the elements level, and the interaction level. There are also other works based on SCOR operation reference model to build supply chains for special purposes [Fayez et al., 2005] [Haller et al., 2008] [Yiqing et al., 2009]. All the approaches above adopt SCOR model,

\footnotetext{
${ }^{3}$ http://www.productivityapex.com/products/scor.asp
} 
which is one of the most known supply chain operation reference models currently, as a base for supply chain process definition and configuration. This reference model could be considered as a standardization of domain knowledge by providing categorized concepts, to act a candidate solution for interoperability problem.

However, all these researches are mainly focusing on supply chain process definition and configuration. But supply chain is not only just a process, also involves product, human, resources etc. The change of manufacturing pattern was one of considerable reasons leaded to supply chain management emergence. Such change was actually due to product complexity, product module reuse extensively. Related works [Vegetti et al., 2005] [Terzi et al., 2007] [Tursi et al., 2009] [Zdravković, 2009] demonstrated that, while product is the centred value of enterprises processes, its information-based model may act as a common pivotal information system to make all enterprise systems interoperating. Hence product plays an important role in supply chain interaction. And it is much more meaningful to considerate them, especially product design and manufacturing stage of product life cycle, for systems integration within extended supply chain environment.

\section{SCOR operation reference model and product ontology}

In the context of networked enterprises, and mainly in supply chain environment in which both business enterprises and manufacturing enterprises are involved, information exchange emphasizes more about inter-enterprise relationships, which are not concerning only products but also processes related to customers, market, service and so on. Information exchange focus is moving from integrated intra-enterprise application packages to internet-based and inter-enterprise application software. Improving Supply Chain Management (SCM) and Customer Relationships Management (CRM) are key processes to enable enterprise value chain [Kirchmer, 2004]. Thus in order to reach maximum comprehension between 
enterprises, and more effective information exchange, more knowledge is needed. Then we introduce SCOR operation reference model and ONTO-PDM product ontology.

\subsection{SCOR operation reference model}

Supply-Chain Operations Reference-model (SCOR) is a process reference model developed and endorsed by the Supply-Chain Council (SCC) [SCC, 2001]. The SCOR model provides a unique framework that links business processes, metrics, best practices and technology features into a unified structure to support communication among supply chain partners and to improve the effectiveness of supply chain management and related supply chain improvement activities. SCOR is based on five distinct management processes: plan, source, make, deliver, and return, as show in Figure 1.Viewed from its structure, it has a pyramid decomposition oriented process detail. Level 1 defines five process types mentioned before as top level. Level 2 is a configuration level, and further describes each process types as process categories, such as source stocked product, source make-to-order product, etc. Level 3 presents detailed process element information for each process category in Level 2.This level includes process flows, all inputs and outputs information, and also performance metrics and best practices for supply chain evaluation. The Council has focused on three process levels and does not attempt to prescribe how a particular organization should conduct its business or tailor its systems/information flow. So Level 4 is not included in SCOR scope, which is used for companies to implement its own SC process flow. 


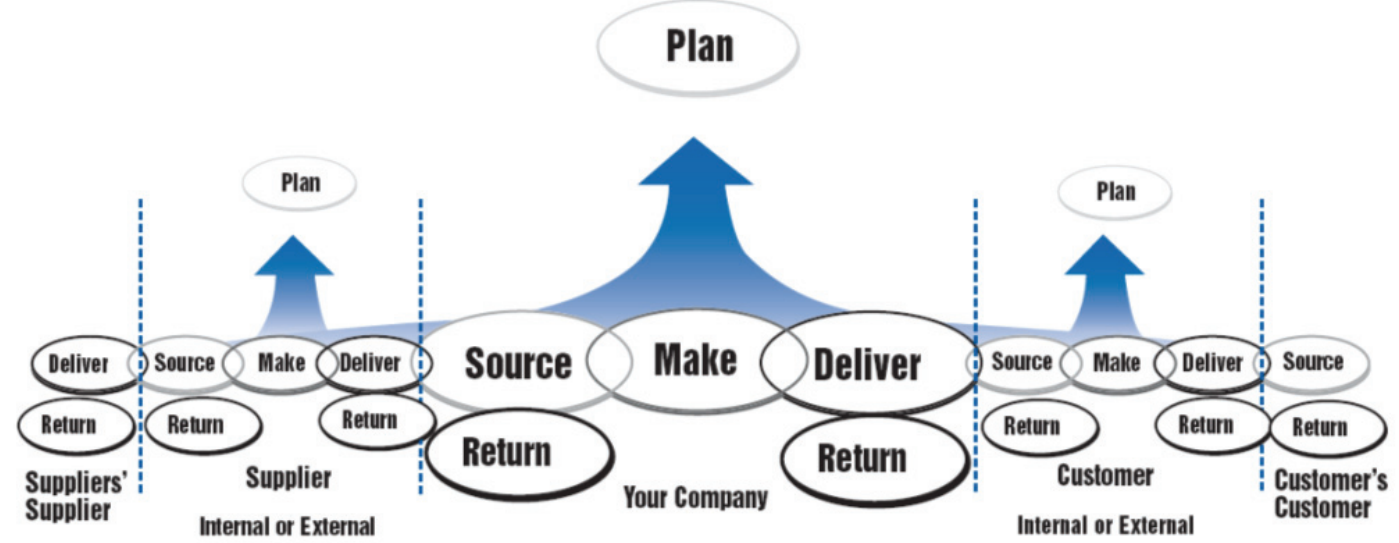

Figure 1 the basic management processes of SCOR operation reference model [SCC, 2001]

SCOR is designed for effective communication among supply chain partners. As a standard language, SCOR model helps SCM (Supply Chain Management), while as an industry standard it also facilitates inter and intra supply chain collaboration, horizontal process integration, by explaining the relationships between processes (i.e., Plan-Source, Plan-Make, etc.) [SCC, 2001]. It specifies the following information:

- all customer interactions, from order entry through paid invoice.

- all physical material transactions, from supplier's supplier to customer's customer, including equipment, supplies, spare parts, bulk product, software, etc.

- all market interactions, from the understanding of aggregate demand to the fulfilment of each order.

- standardised process configuration model for supply chain process modelling

- descriptions of best practices related to each process

- standard measure metric for performance measures

\subsection{Product ontology (ONTO-PDM)}

Tursi et al. [Tursi et al., 2009] have worked on the product-centric information system interoperability in networked manufacturing enterprises, and proposed a Product Ontology, the ONTO-PDM, for Product Data Management and interoperability. This integrated and common model formalizes the knowledge related to product data management at the business and the manufacturing levels of enterprises (B2M, Business to Manufacturing), in order to achieve the interoperability between systems. Fig. 2 shows an extract of the ONTO-PDM ontology concepts. It adopts two standards: the IEC 62264 [IEC, 2002] and the ISO 10303 
STEP-PDM [STEP, 2004]. The IEC 62264 set of standards define an information exchange framework to facilitate the integration of business applications and manufacturing control applications, which related to product production phase. It can be used to integrate business enterprise applications such as ERP, supply chain management system with manufacturing enterprise applications such as MES. STEP-PDM is a standard for computer interpretable representation and exchange of product definition data, which aims at providing a mechanism capable of describing product data throughout the life cycle of a product. It is used for exchanging information between ERP and PDM systems. It deals with typical product-related information including geometry, engineering drawings, project plans, part files, assembly diagrams, numerical control machine-tool programs, analysis results, correspondence, bills of material, engineering change orders and many more [IEC, 2002]. So ONTO-PDM concentrates most but not all product technical and geometrical data and business related information span from its development to its manufacturing and related business processes. 


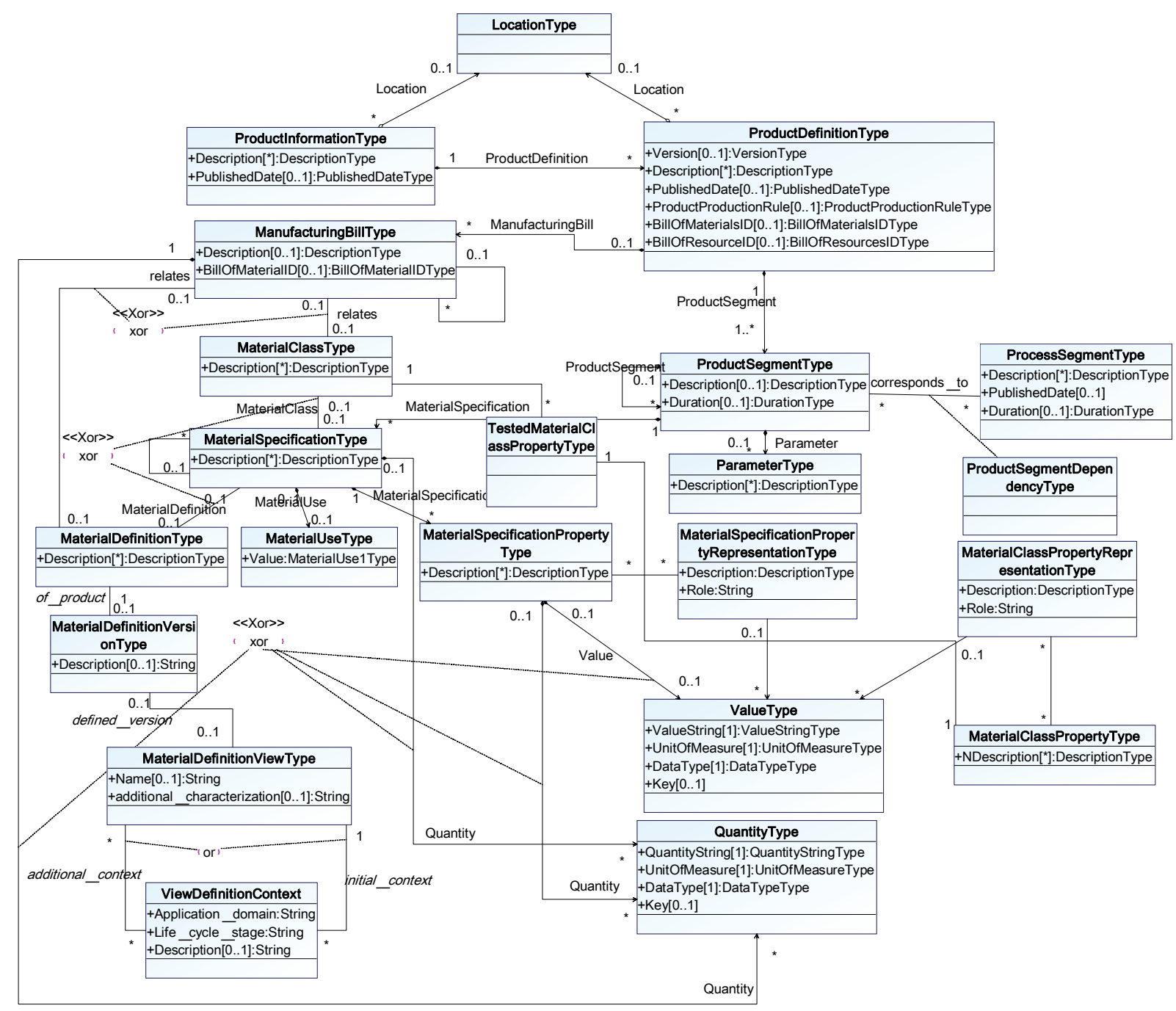

Figure 2 An extract of the ONTO-PDM Product Ontology [Tursi et al., 2009]

\section{A product-centric supply chain ontology framework for networked enterprises interoperability}

\subsection{Framework of product-centric supply chain ontology}

As presented previously, the SCOR operation reference model focuses on supply chain processed, and enables reducing the difficulties in supply chain implementation by providing a structured process model, standard workflows, and a series of measurement. Meanwhile its scope covers customer interactions, physical product transactions, market interactions. However it does not attempt to describe every business processes or activities including the data flows between them. Specifically, the model does not address: sales and marketing (demand generation), product development, research and development, and some elements of 
post-delivery customer support. It is important to note that this model describes processes not functions. In other words, the model focuses on the activities involved not the person or organizational elements that perform these activities [SCC, 2001]. Furthermore, SCOR does not take into account the detailed product information shared among all stakeholders in the supply chain. ONTO-PDM just plays this missing role, addressing the scope facing engineering to business from product design to product manufacturing. Even more, it deals with the process functions by defining personnel, equipments or organizations to support the processes. The SCOR operation reference model and ONTO-PDM are then each other complementary for representing and sharing information in a supply chain network environment. As mentioned above, ONTO-PDM and SCOR operation reference model are promising candidates for building such supply chain ontology framework for networked enterprises interoperability.

Then, focusing on information flows in supply chain, we are proposing a productcentric supply chain ontology framework for networked enterprises, defining a common share model for product data exchange, by aligning product ontology with SCOR operation reference model, mainly, all processes that have relationships with the product in a supply chain, in which the shared knowledge concerns not only the product design and production, but also the product repair, the product return, the warehouse management, and related customers activities, as summarized in Figure. 3. 


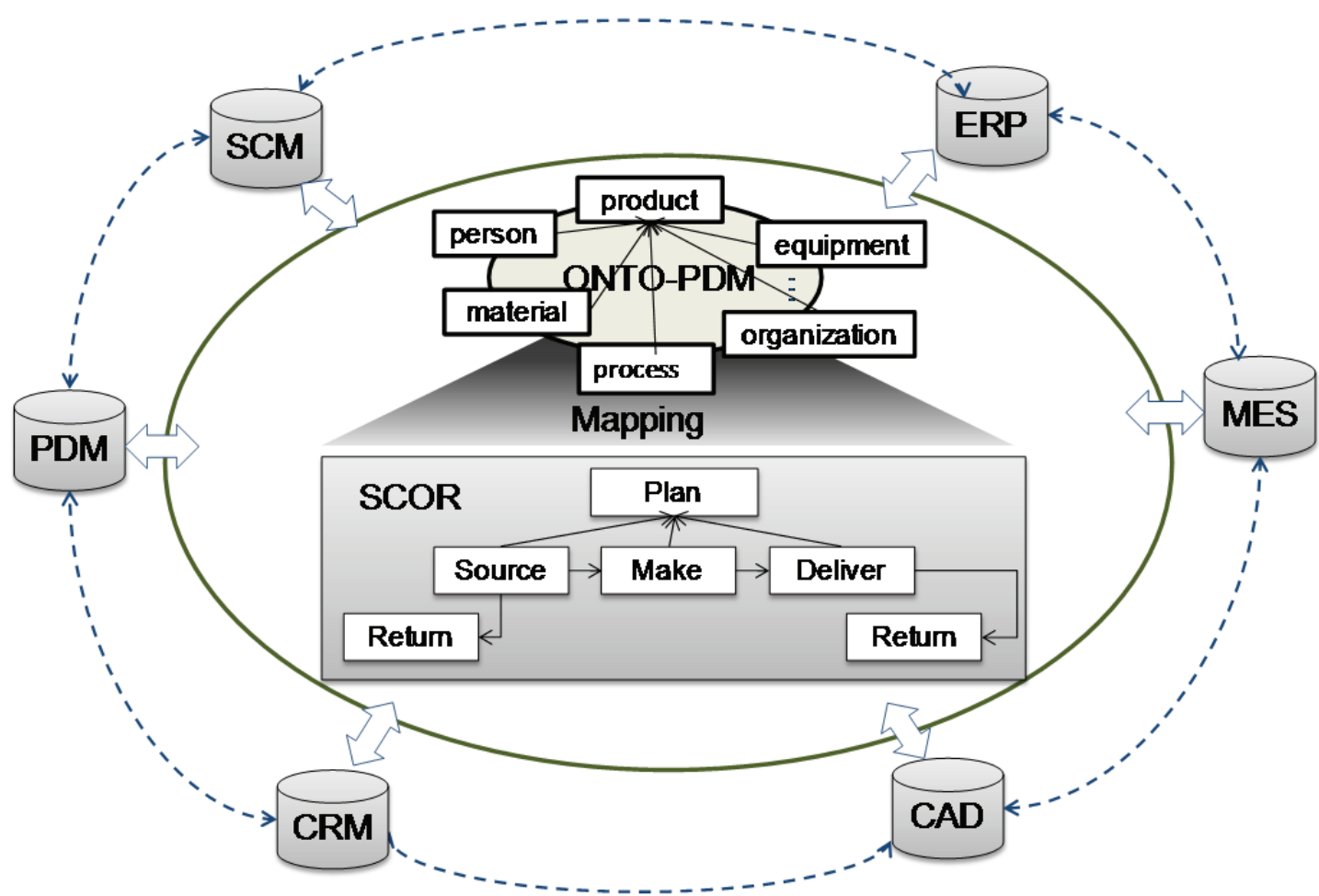

Figure 3 Framework of Supply Chain ontology for networked enterprises interoperability.

As shown on Figure3, the Supply Chain ontology is composed of SCOR ontology and the ONTO-PDM, linked through semantic mappings. The ONTO-PDM defines entities related to product engineering and manufacturing, for example product, equipment, material, person, process and so on. I also formalises the definitions about processes, which could be used for expressing information linking business systems with manufacturing systems. From a supply chain perspective, products can also be considered as main objects with all the corresponding properties related to ordering, producing, delivering, and returning. Besides, the SCOR ontology defines not only interacted activities between suppliers, customers and market, but also metrics used to better formalize supply chain process with close relation to SCM.

\subsection{Formalizing an Ontology based on SCOR}

Our work focus on information flow among networked enterprises in supply chain environment, nevertheless the current SCOR operation reference model configures process focus on its physical supply chain process flow, not integration yet an "information view" 
[Millet et al., 2009]. So we are trying to formalize an Ontology based on SCOR operation reference model to enrich it with semantic meanings, in order to align the supply chain process within networked enterprises and facilitate interoperation. We take the Supply Chain Reference Model Version 7.0 as a main concepts and structures bases and we refer to the SCOR-KOS OWL Model proposed by [Zdravković M., 2010] which has been designed for rapid supply chain process configuration. These modelling activities are executed in the environment of Protégé $4.1^{4}$ using OWL DL as the modelling language.

Let us give an overview of SCOR-KOS OWL model proposed by [Zdravković M., 2010]. It includes classified input and output concepts supporting supply chain processes, processes structure and benchmark, and system related entities. In our context, with the objective of aligning information systems models, we are focusing on information exchange between SCOR processes. Thus we reused only part of the SCOR-KOS model related to the definition of input and output entities in SCOR processes (Figure 4b). However, reusing the initial SCOR-KOS ontology structure, we analysed the SCOR operation reference model processes and extended the definition of some SCOR-KOS concepts related to the type and the category processes (Figure 4a). This ontology update is justified in order to take into account the specific relationships and information flows between each kind of processes in a networked supply chain. Our SCOR ontology includes the three process levels of SCOR (process type, process category, process element), all input and output concepts in relationships with processes, performance attributes, best practices, and features (Figure 4a).

\footnotetext{
${ }^{4}$ http://protege.stanford.edu/
} 


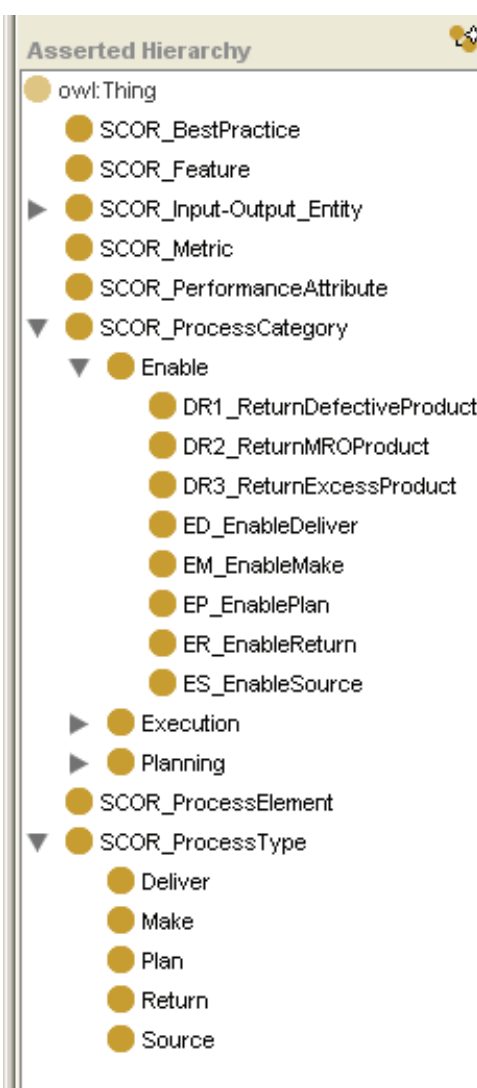

(a)

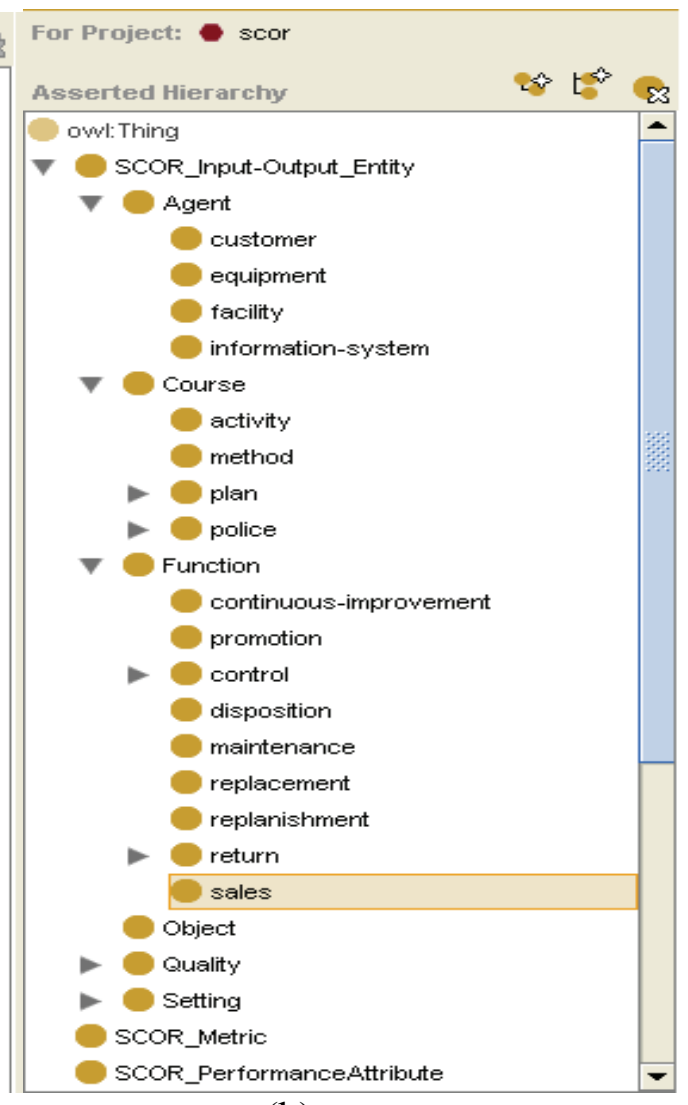

(b)

Figure 4 Fragment of SCOR ontology related to (a) SCOR processes (b) SCOR-KOS Input/output entities [Zdravković M., 2010]

As mentioned before, SCOR operation reference model's scope standardises three process levels. SCOR_ProcessType represents the processes at level 1, including plan, source, make, deliver, return. SCOR_ProcessCategory represents level 2 processes' types: Planning(includingP1_PlanSupplyChain,P2_PlanSource,P3_PlanMake,P4_PlanDeliver,P5_ PlanReturn), Execution(such as D1_DeliverStockedProduct,M1_Make-toStock,S1_SourceStockedProduct,SR1_ReturnDefectiveProduct, etc), Enable(such as DR1_ReturnDefectiveProduct, ED_EnableDeliver, EM_EnableMake,ER_EnableReturn, etc). SCOR_ProcessElement formalises the detail process element information for each Level 2 SCOR_ProcessCategory. Even if the SCOR operation reference model requires that any company should decompose its processes to more detailed levels (4 or more), we have limited our ontology to the level 3, because in a networked supply chain, the boundaries of the 
information flows are related only to interactions between companies. Thus, SCOR_ProcessElement is defined as the smallest process unit in our SCOR ontology.

The SCOR ontology is represented by OWL DL, having explicit conceptual meaning, consistent and precise semantics. The ontology language OWL DL is based on Description logics (DL), which is a family of formal knowledge representation languages [Baader et al. 2003]. The above ontology model can be expressed by the following equivalent axioms in DL for example.

Axiom1. All values of the has_processcategory of the SCOR_ProcessType class is the member of the class SCOR_ProcessCategory.

\section{SCOR_ProcessType $\sqsubseteq \forall$ has_processcategory.SCOR_ProcessCategory}

Axiom2. All value of the has_processelement of the SCOR_ProcessCategory class is the member of the class SCOR_ProcessElement.

\section{SCOR_ProcessCategory $\sqsubseteq \forall$ has_processelement.SCOR_ProcessElement}

Axiom3. All values of the has_inputand has_output of the SCOR_ProcessElementare members of the class SCOR_InputOutput_Entity

SCOR_ProcessElement $\sqsubseteq \forall$ has_input.SCOR_InputOutput_Entity

SCOR_ProcessElement $\subseteq \forall$ has_output.SCOR_InputOutput_Entity

Axiom4. All value of the connectBy SCOR_ProcessElementis a member of itself. $S C O R_{-}$ProcessElement $\subseteq \forall$ connectBy.SCOR_ProcessElement

Axiom5. The object property is_configured_of is an inverse of the has_processcategory object property.

$$
\text { is_configured_of } \equiv \text { has_processcategory }{ }^{-}
$$

Axiom6. The class Plan is subclass of SCOR_ProcessType class

$$
\text { Plan } \sqsubseteq \text { SCOR_ProcessType }
$$

\subsection{Alignment between SCOR and ONTO-PDM}

The SCOR Ontology and ONTO-PDM have been developed separately. In order to obtain a common share supply chain ontology framework, some correspondence mappings must be established between them. As the SCOR operation reference model, in some extent, is still a conceptual model, there are not too many properties and relationships have been 
defined yet. Besides, some of the concepts are not fully standardised, such as feature, information input and output, etc. Thus our mapping is based only at conceptual level. In order to make the two ontologies interactive, we are following the steps below:

(1) Identify common concepts between ONTO-PDM and SCOR

(2) Formalise these concepts with DL

(3) Identify mapping rules

(4) Compute inference concepts

(5) Concepts alignment

\section{Identify common concepts between ONTO-PDM and SCOR (1)}

Reviewing the content of SCOR Ontology and ONTO-PDM, although they are two separate ontologies concerning different aspects, SCOR Ontology is specifically used for capturing complex supply chain management process into a standard process model, while ONTO-PDM is designed for integrating all relevant information related to products lifecycle. However, there are still some common concepts between them. ONTO-PDM defines product related information in the phase of design and manufacturing. Sensibly, some linguistic and terminological commons also exist. We matched the entities of ONTO-PDM with the inputs and outputs, and processes concepts of SCOR Ontology and we formalised each concept with DL expressions. Table 1 lists a fragment of relevant concepts that have semantic correspondences.

Table 1. Fragment of relevant concepts between ONTO-PDM and SCOR.

\begin{tabular}{l|l}
\hline \multicolumn{1}{c|}{ Entity of ONTO-PDM } & \multicolumn{1}{c}{ Concept of SCOR } \\
\hline $\begin{array}{l}\text { ProcessSegmentType/ } \\
\text { ProductSegmentType }\end{array}$ & SCOR_ProcessElement \\
\hline $\begin{array}{l}\text { EquipmentSpecificationType/ } \\
\text { EquipmentType }\end{array}$ & Equipment \\
\hline EquipmentlRequirementType & Equipment-and-facilities-schedule \\
\hline $\begin{array}{l}\text { ProductDefinitionType/ } \\
\text { ProductInformationType }\end{array}$ & Product \\
\hline $\begin{array}{l}\text { PersonnelSpecificType/ } \\
\text { PersonType }\end{array}$ & customer \\
\hline ProductionCapabilityType & production-capability \\
\hline ProductionScheduleType & production-schedule \\
\hline ProductionPerformanceType & performance-plan \\
\hline ProductionPerformanceType & $\begin{array}{l}\text { performance-plan/ } \\
\text { goal-performance-plan }\end{array}$ \\
\hline MaintenanceInformationType & maintenance \\
\hline
\end{tabular}


Formalise these concepts with DL (2)

Table 2 shows the formalized DL expression of the entity ProcessSegmentType /ProductSegementType of ONTO-PDM and the concept SCOR_ProcessElement of SCOR Ontology.

Table 2. DL formal expression of concepts

ONTO-PDM: ProcessSegmentType/ProductSegmentType (defined by the attributes and the relationship between ProcessSegmentType and ProductSegmentType)

ProcessSegmentType $\subseteq \forall$ Description. DescriptionType ProcessSegmentType $\sqsubseteq \forall$ Duration.DurationType ProductSegmentType $\sqsubseteq \forall$ Description. DescriptionType ProductSegmentType $\subseteq \forall$ Duration. DurationType ProcessSegmentType $\sqsubseteq \forall$ correspond_to.ProductSegmentType ProcessSegmentType $\subseteq \forall i s_{-}$composed_of.ProcessSegmentType

SCOR: SCOR_ProcessElement (defined as subprocess of SCOR_ProcessCategory and has input, output)

SCOR_ProcessCategory $\subseteq \forall$ has_processelement.SCOR_ProcessElement $S C O R_{-}$ProcessElement $\subseteq \forall$ connectBy.SCOR_ProcessElement SCOR_ProcessElement $\subseteq \forall$ has_input.SCOR_InputOutput_Entity SCOR_ProcessElement $\sqsubseteq \forall$ has_output.SCOR_InputOutput_Entity

\section{Identify mapping rules (3)}

Identifying mappings rules is still an open issue. Many researchers have been done to try to compute automatically or semi-automatically these rules, in different domains [Kalfoglou et al. 2003]. This issue is out of the scope of this work. In our work, we adopt SWRL to describe mapping rules. SWRL is proposed as a semantic web rule language combining OWL DL and RuleML (Rule Markup Language) [Horrocks I., 2004] SWRL rules are really a kind of OWL axiom. They can make for more expressive property and class axioms [Parsia B, 2007], and be applied to semantic transformation within inference engines

We then postulate some mapping rules between concepts from both ontologies.

Rule 1.

SCOR:SCOR_InputOutput_Entity (? y) ,SCOR_InputOutput_Entity (? z), SCOR_ProcessElement (? $x 1)$, SCOR_ProcessElement $(? x 2)$, has_input $(? x 1, ? y)$, has_output $(? x, ? z)$, proceed_by $(? x 1, ? x 2)$ 
Rule 2.

Rule 3.

SCOR:SCOR_(InputOutput_Entity(? y) ), SCOR_ProcessElement(? $x 1)$,

SCOR_ProcessElement $(? x 2)$, has_output $(? x 1, ? y)$, proceed_by $(? x 1, ? x 2)$ $\rightarrow$ ONTO: ProcessSegmentType $(? \times 1)$

Rule 4.

$$
\begin{gathered}
\text { SCOR:SCOR_(InputOutput_Entity(?y) ),SCOR_ProcessElement }(? \times 1), \\
\text { SCOR_ProcessElement }(? \times 2), \text { has_input }(? \times 1, ? y), \text { proceed_by }(? \times 1, ? \times 2) \\
\rightarrow \text { ONTO:ProcessSegmentType }(? \times 1)
\end{gathered}
$$

Rule 5.

ONTO: ProductDefinitionType(? $x)$, ProductSegmentType(?y), has_productsegment $(? x, ? y)$ $\rightarrow$ SCOR:Production_Plan $(? x)$

ONTO: ProductionScheduleType(?x), ProductionRequestType(?y), has_productrequest(? $x, ? y)$ $\rightarrow$ SCOR:Production_Schedule(? $x)$

The Rules 1,2, and 3 describe if entity SCOR_ProcessElement in SCOR ontology has either input or output, or both can infer to be equivalent to entity ProcessSegmentType of ONTO-PDM product ontology. Similarly, Rule 4 and Rule 5 are also conditional inference rules, indicate entity ProductDefinitionType and ProdcutionScheduleType need to satisfy certain properties which could then be mapped to Production_Plan and Production_Schedule separately

\section{Compute inference concepts (4) and Concepts alignment (5)}

Using Pellet ${ }^{5}$ inference engine, we are able to verify the coherency of all merged ONTO-PDM and SCOR DL expressions including the SWRL mapping rules. Pellet is also able to reclassify those concepts and, thus, identifying concepts candidates for being aligned. From Pellet results, we are then able to reconstruct the ontology taking into account the alignments suggested by Pellet. The resulting ontology framework, the product-centric supply chain ontology framework for networked enterprises interoperability, is then resulting from an alignment of both ONTO-PDM and SCOR ontologies, taking into account of mapping rules.

\subsection{A Scenario example}

In order to show how these steps above take place and how this product-centric supply chain ontology framework works, we consider an example from the main body manufacturing

\footnotetext{
${ }^{5} \mathrm{http}: / /$ clarkparsia.com/pellet
} 
process of double column $\mathrm{CNC}$ guideway \& surface grinding machine in Hangzhou Machine Tool Company. As grinding machine is usually made by order, we take account of SCOR Make-to-Order (level 3) process.

The SCOR process make-to-order.

This process of manufacturing in a make to order environment adds value to products through mixing, separating, forming, machining, and chemical processes. A make to order environment is one in which products are completed after receipt of a customer order and are built or configured only in response to a customer order Then we configure make to order supply chain based on SCOR reference model, and extend it according to real producing process (level 4)(Figure 5).

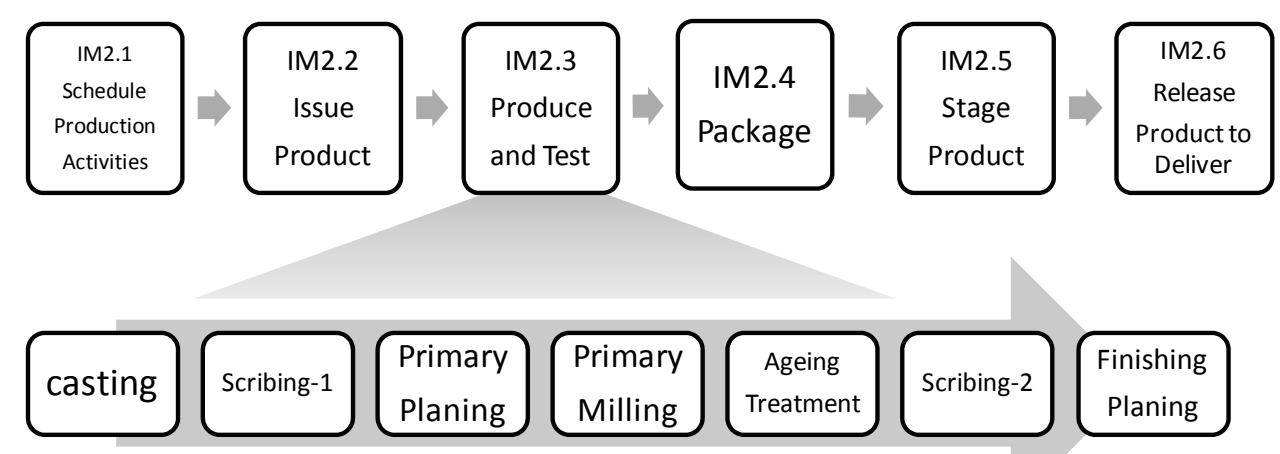

Figure $5 \mathrm{~A}$ detailed make-to-order process element of main body production

In this supply chain process, several enterprise systems are involved inside, for instance, ERP managing order, sourcing, EBOM, customer information, ProE for designing, CAPP for producing routing, etc, In order to make these systems interaction, we build an instance of make-to-order process IM2.1 to IM2.6 in SCOR ontology, define inputs and outputs elements according to process standard structure in SCOR operation reference model, seeing Figure 6(a).After using the Pellet inference engine with SWRL rules defined before, the inferred results indicate that IM2.1 is an instance of SCOR_ProcessElement an also an instance of ProcessSegmentType (Figure 6(b), Figure 7). 


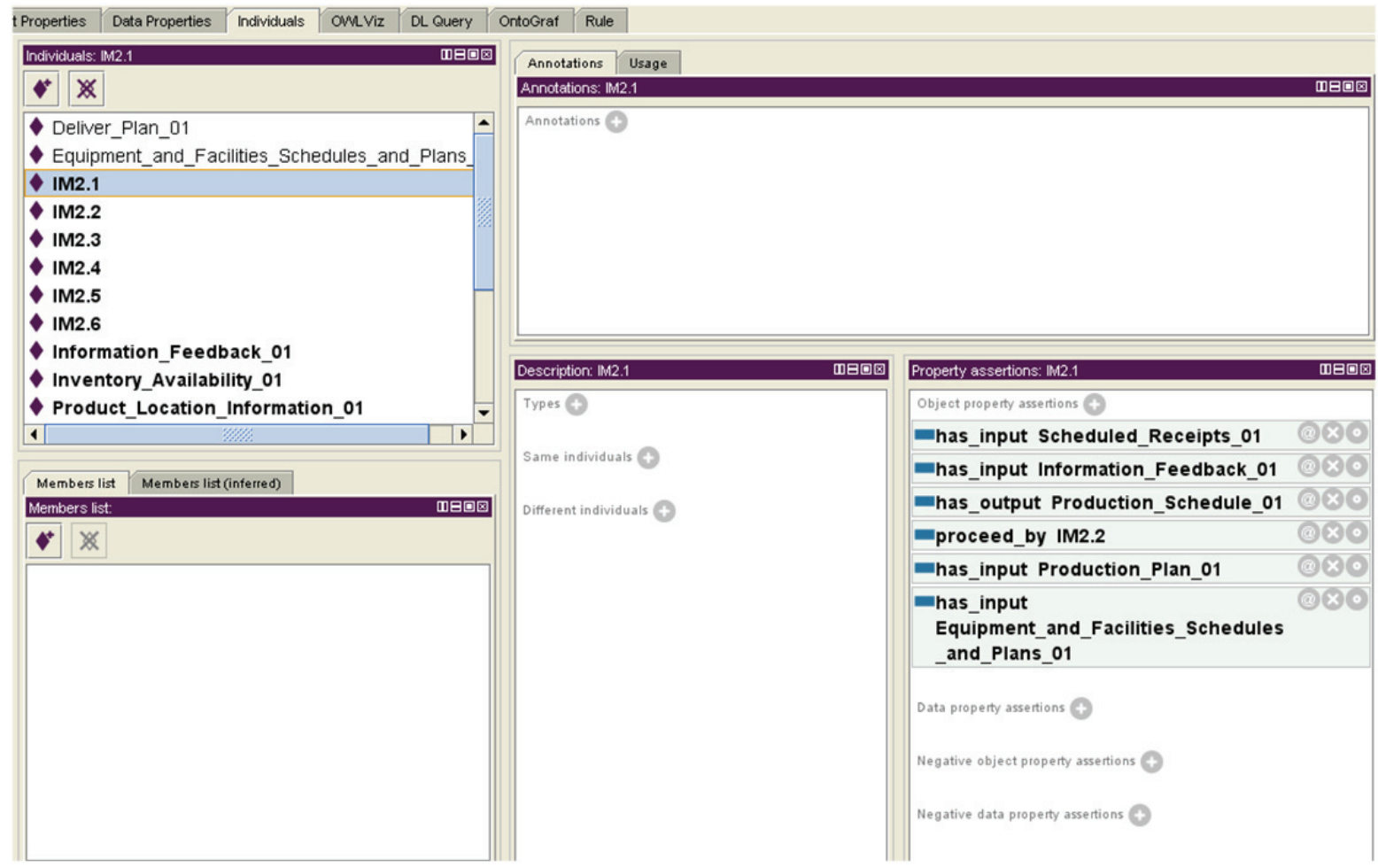

Figure 6a Instance of Make-to-order Process in Protégé

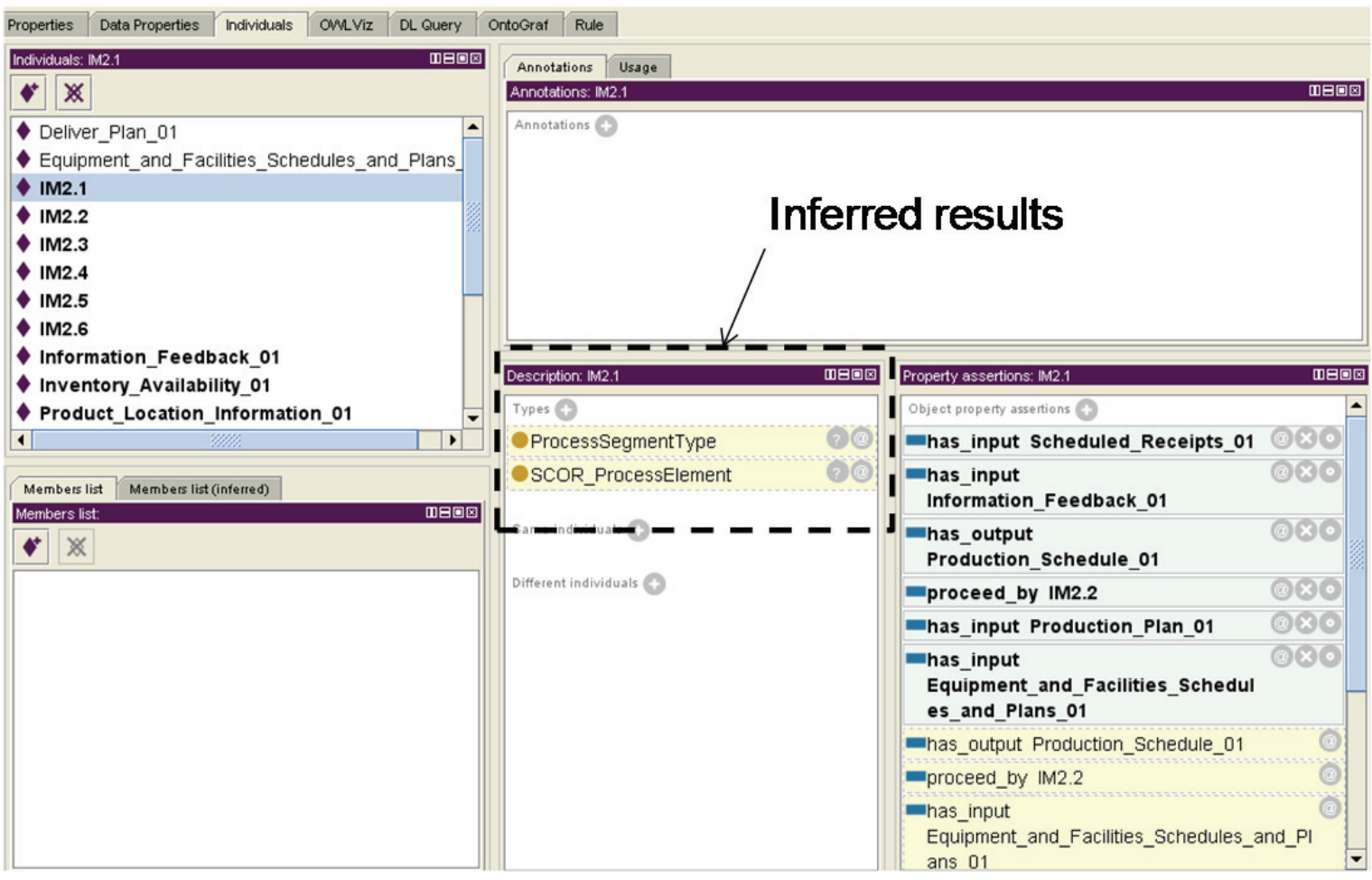

Figure $6 \mathrm{~b}$ Inferred results by pellet 


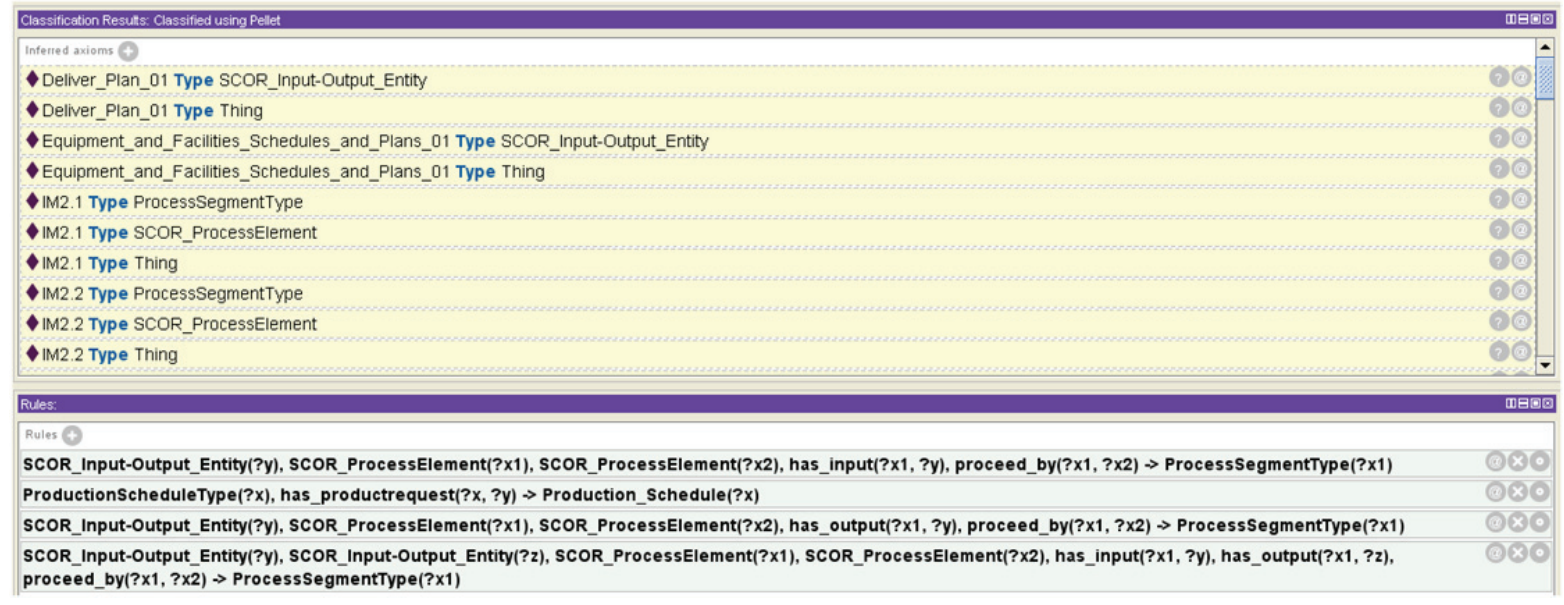

Figure 7 SWRL rules and more inferred results in Protégé

According to the pellet inference results above, we align SCOR ontology with ONTO-

PDM. This integrated model includes both information of supply chain and manufacturing

domain. Seeing in Figure 8, the right part inside the dash line is from ONTO-PDM, indicating material, equipment, producing information, etc.

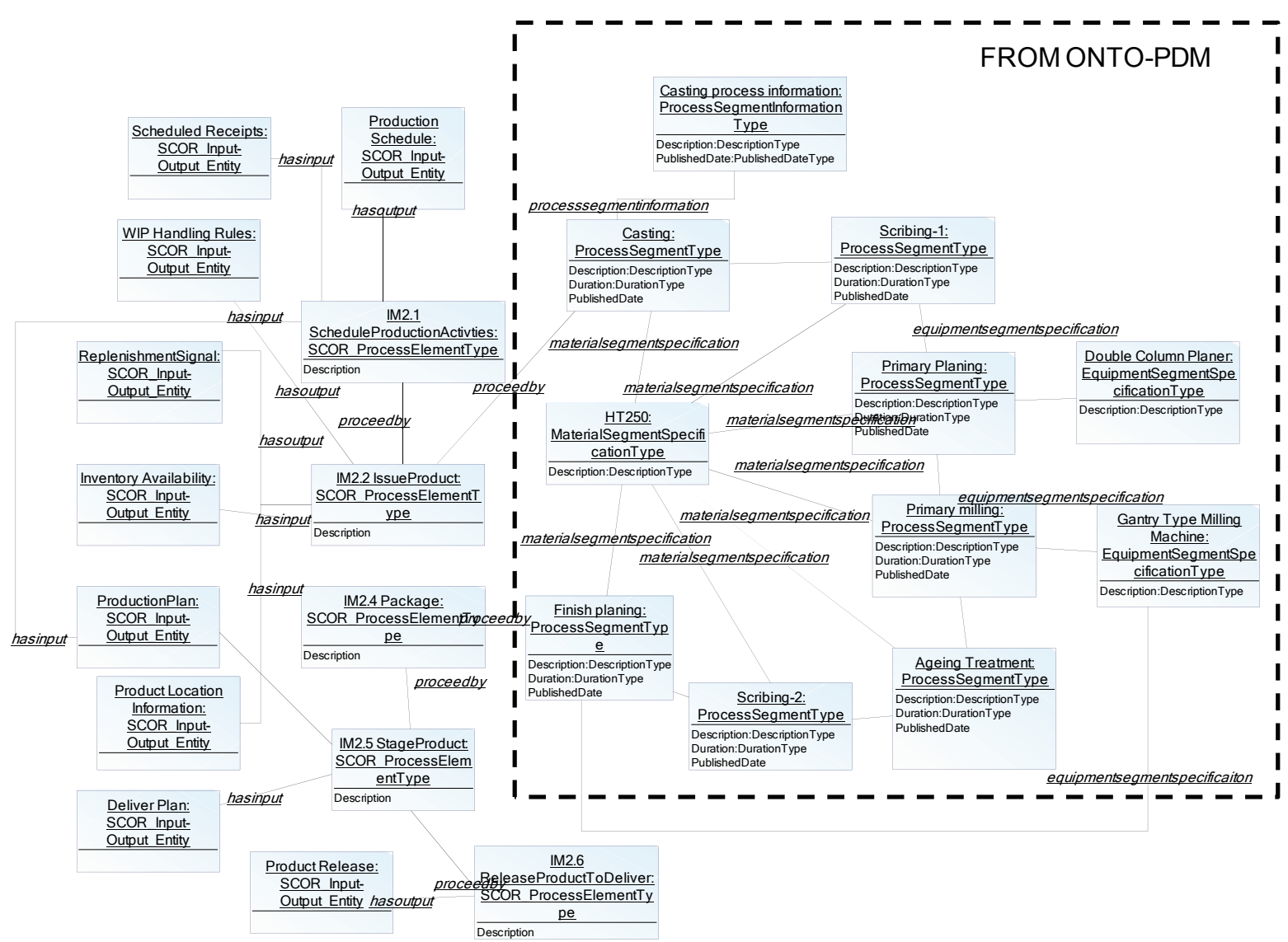

Figure 8 UML fragment of make-to-order process element of main body production 


\section{Conclusion and Future work}

Systems interoperability is still a key issue of importance today andis a critical factor for enterprise to manage and improve its value chain in global competition. In this paper, we have proposed a common shared ontology framework for networked enterprises information systems interoperability in a supply chain environment. After establishing our problem context and approach for networked enterprise information systems interoperability, we studied the SCOR operation reference model and ONTO-PDM from their scope, purpose, content, structure etc. in order to make sure that they are qualified for building a common shared ontology framework. Subsequently, an ontology based engineering approach was proposed for identifying concepts alignments in order to propose a share ontology as the reference information model for applications interoperability in a supply chain context. This core common ontology consists of an integration of SCOR ontology and ONTO-PDM with SWRL rules, and is formalized using Description Logic to assure its coherency and completeness.

The common shared ontology framework integrates the supply chain management aspect with product design, manufacturing engineering aspect, reaching a more range of interoperability than traditional supply chain. This Standard based Ontology represented by web ontology language, which is more exploitable for networked systems integration. Meanwhile, this work has to be extended in order to take into account other SCOR processes and associated flows. As the SCC is still making effort on SCOR operation reference model changing and completing, our SCOR ontology has to be also continuously developed and further refined as well. The issue of ontology change management can then be viewed as an open problem with the need for additional research to address related issues For example, many of the inputs/outputs concepts, best practise defined in SCOR, etc.have not been formalised (they are still described by a free text), which are impediments to formalizing the 
SCOR ontology. Building and implementing ontology is an important but time consuming effort; other related issues which need to be investigated include being able to calculate the real Return of Investments (ROI) in practical industrial contexts.

Acknowledgements: Our work is supported by Chinese Science and Technology Support Plan Project (2011BAB02B01), Chinese National Natural Science Foundation Project (51175463)(61175125), and Zhejiang Provincial Natural Science Foundation Project (Y1110414 ).

\section{References}

Baader F., Calvanese D., McGuinness D. L., Nardi D., Patel-Schneider P. F.,2003. The Description Logic Handbook: Theory, Implementation, Applications. Cambridge University Press, Cambridge, UK, Fayez, M., Rabelo, L., Mollaghasemi, M., 2005. Ontologies for supply chain simulation modeling. In: 37th Conference on Winter Simulation Conference, 4-7 December 2005.Orlando, Florida.

Gruber T.R,1993.A translation approach to portableontology specifications. Knowledge Acquisition,5(2),199-229.

Haller A., GontarczykJ., and Kotinurm P., 2008. Towards a complete SCM ontology: the case of ontologising RosettaNet. In Proceedings of the 2008 ACM Symposium on Applied Computing,SAC '08Fortaleza, Ceara, Brazil, March 16 - 20, 2008). 1467-1473.

Horrocks I., Patel-Schneider P.F., Boley H. et al.,2004. SWRL: A Semantic Web Rule Language Combining OWL and RuleML.W3C Member Submission 21 May 2004. Available from: http://www.w3.org/Submission/SWRL/.

IEEE, 1990.Institute of Electrical and Electronics Engineers: IEEE Standard Computer Dictionary: A Compilation of IEEE Standard Computer Glossaries. New York, NY (1990)

IEC,2002. Enterprise-control system integration. Part 1. Models and terminology. Part 2:

Model objectattributes: ISO/IEC FDIS Standard, IEC and ISO, Geneva, Switzerland Jagdev, H. S., Thoben, K. D., 2001. Anatomy of enterprise collaborations. Production Planning and Control, 12/5, 437-451.

Kalfoglou K., Schorlemmer M., 2003. Ontology mapping: the state of art. The Knowledge Engineering Reviews, 18(1), 1-31.

Kirchmer, M., 2004. E-business process networks - successful value chains through standards. Journal of Enterprise Information Management, 17(1), 2-30. 
Kollberg, M. and Dreyer, H., 2006. Exploring the impact of ICT on integration in supply chain control: a research model. In: Proceedings of the 13th International EurOMA Conference "Moving up the Value Chain", Glasgow, UK, 18-21 Jun.2006

Millet, P.A., Schmitt, P. and Botta-Genoulaz, V., 2009. The SCOR model for the alignment of business processes and information systems. Enterprise Information Systems, 3(4), $393-407$.

Parsia B,, 2007. Understanding SWRL (Part 1). Available from: http://weblog.clarkparsia.com/2007/08/12/understanding-swrl-part-1/.

Sakka, O., Millet, P.A., and Botta-Genoulaz, V.,2010. A OWL based ontology of SCOR model : a prerequisitie for strategic alignment. In: Proceedings of $8^{\text {th }}$ International Conference of Modeling and Simulation (MOSIM'10), 10-12 May 2010, Hammamet, Tunisia.

Sheer, A. W., Schneider K. 2005. ARIS architecture of integrated Information Systems.Handbook of Information Systems, Springer, Berlin

STEP,2004.ISO/TS 10303 STEP modules related to Product Data Management: Industrial automation systems and integration - Product data representation and exchange, Geneva

Stanley E. Fawcett,2001. Achieving World-Class Supply Chain Alignment:Benefits, Barriers, and Bridges Center for Advanced Purchasing Studies. Arizona: Tempe.

SCC,2001. Supply chain operations reference model 7.0.Supply Chain Council. Available from:http://www.supply-chain.org

Terzi, S., Panetto, H., Morel, G., Garetti, M.,2007.A holonic metamodel for product lifecycle management. International Journal of Product Lifecycle Management, 2(3), 253-289.

Tursi A., 2009. Ontology-based approach for enterprise production systems interoperability, $\mathrm{PhD}$, University Henri Poincaré Nancy I, Politecnico di Bari, November

Tursi A., Panetto H., Morel G., Dassisti M.,2009. Ontological approach for Products-Centric Information System Interoperability in Networked Manufacturing Enterprises. IFAC Annual Reviews in Control. 33(2), 238-245,.

Vegetti, M., Henning, G. P., Leone, H. P.,2005. Product ontology: definition of an ontology for the complex product modelling domain. In:4th Mercosur Congress On Process Systems Engineering, Rio de Janeiro,Brazil.

Vujasinovic M.,IvezicN., KulvatunyouB. et al, 2009.A semantic-mediation architecture for interoperable supply-chain applications.International Journal of Computer Integrated Manufacturing, 22(6), 549-561. 
Yiqing, L., Lu, L., Chen, L,2009. Decision-Making for Supplier Selection Based on Ontology and Rules. In: 2nd International Conference on Intelligent Computation Technology and Automation,. Washington, DC, USA.

Zdravković, M., Trajanović, M.,2009. Integrated Product Ontologies for Inter-Organizational Networks. Computer Science and Information Systems, 6/2, 29-46.

Zdravković, M., Panetto, H., Trajanović, M.,2010. Concept of semantic information pool for manufacturing supply networks. International Journal of Total Quality Management and Excellence. 37( 3), 69-74 .

Zdravković, M., Panetto, H., Trajanović, M. 2010. Towards an Approach for Formalizing the Supply Chain Operations. In: 6th International Conference on Semantic Systems, ACM ICP, $8^{\text {th }}$ Jun,2010 Graz, Austria. 